\title{
Infralimbic GluN2A-Containing NMDA Receptors Modulate Reconsolidation of Cocaine Self-Administration Memory
}

\author{
Madalyn Hafenbreidel', Carolynn Rafa Todd' and Devin Mueller*,1,2 \\ 'Department of Psychology, University of Wisconsin-Milwaukee, Milwaukee, WI, USA; '2Department of Basic Sciences, Ponce Health Sciences \\ University-School of Medicine/Ponce Research Institute, Ponce, Puerto Rico
}

\begin{abstract}
Addiction is characterized by high relapse susceptibility, and relapse can be triggered by drug-associated cues. Cue presentation induces retrieval of the drug-cue memory, which becomes labile and must be reconsolidated into long-term storage. Repeated unpaired cue presentation, however, promotes extinction. Cue-reactivity can be reduced by blocking reconsolidation or facilitating extinction, which are mediated by NMDA receptors (NMDArs). However, the role of NMDArs in either process following self-administration is unclear. Thus, to determine their role in extinction, rats learned to self-administer cocaine before receiving injections of the NMDAr antagonist CPP immediately after four 45-min extinction sessions. During a subsequent 90-min extinction retention test, CPP-treated rats lever pressed less than saline-treated rats indicating that NMDAr blockade facilitated extinction or disrupted drug-cue memory reconsolidation. In addition, infusing CPP into the infralimbic medial prefrontal cortex (IL-mPFC), a structure implicated in extinction, before four 45-min or immediately after four 30min extinction sessions, had similar results during the extinction retention tests. Next, the GluN2A-selective antagonist NVP or GluN2B-selective antagonist Ro25 was infused into IL-mPFC or nucleus accumbens (NAc) shell, another structure implicated in extinction, after four 45-min extinction sessions. Blocking GluN2A-, but not GluN2B-, containing NMDArs, in IL-mPFC or NAc shell reduced lever pressing during the extinction retention tests. Finally, to dissociate reconsolidation from extinction, NVP was infused into IL-mPFC after four I0-min reactivation sessions, which resulted in reduced lever pressing during the retention test. These results indicate that IL-mPFC GluN2A-containing NMDArs modulate reconsolidation, and suggest a novel treatment strategy, as reducing cue reactivity could limit relapse susceptibility.

Neuropsychopharmacology (2017) 42, III3-II25; doi:I0.1038/npp.20 I6.288; published online 25 January 2017
\end{abstract}

\section{INTRODUCTION}

Drug addiction is characterized by compulsive drug seeking and taking, and chronic relapse (McLellan et al, 2000). Addiction is maintained by cues associated with drug administration, as these cues can trigger retrieval of the original drug-cue memory, evoke craving, and promote relapse (Childress et al, 1986). Following memory retrieval, the memory is labile and requires restabilization into longterm storage through a process called reconsolidation (Miller and Marshall, 2005; Misanin et al, 1968; Nader et al, 2000). However, when the cue is repeatedly presented without drug reinforcement, a new inhibitory extinction memory forms (Millan et al, 2011; Quirk and Mueller, 2008). Both reconsolidation and extinction share similar, but distinct, underlying mechanisms (Suzuki et al, 2004; Tronson and Taylor, 2007), with some research suggesting they compete at the molecular level (Nader, 2003). Thus, dissociating these processes is challenging. Behaviorally, they are distinguished

\footnotetext{
*Correspondence: Dr D Mueller, Department of Basic Sciences, Ponce Health Sciences University-School of Medicine, Ponce Research Institute, PO Box 7004, Ponce 00732-7004, Puerto Rico, Tel: + I 787 8402575 Ext. 2588, Fax: + 787844 1980, E-mail: dmueller@psm.edu Received 16 March 2016; revised 10 December 2016; accepted 19 December 2016; accepted article preview online 2 January 2017
}

by the amount of time the animal undergoes a reactivation or extinction session. Brief sessions reactive the original memory, which subsequently undergoes reconsolidation, but fail to result in extinction (reduced responding to the stimulus) as longer sessions would (Nader, 2003; Quirk and Mueller, 2008; Suzuki et al, 2004; Tronson and Taylor, 2007).

One mechanism underlying both extinction consolidation and memory reconsolidation is NMDA receptor (NMDAr) activity (Nader, 2003). NMDArs are necessary for learning and memory (eg, McLamb et al, 1990; Mondadori et al, 1989; Thompson et al, 1987), including extinction of conditioned fear (Liu et al, 2009; Santini et al, 2001; Suzuki et al, 2004) and drug seeking (Hafenbreidel et al, 2014; Hsu and Packard, 2008). Moreover, NMDArs are necessary for reconsolidation, as blocking NMDArs following a short reactivation session disrupts the reconsolidation of conditioned fear (Lee et al, 2006), contextual (Pedreira et al, 2002), appetitive (Lee and Everitt, 2008), odor discrimination (Torras-Garcia et al, 2005), and drug-induced conditioned place preference memories (Alaghband and Marshall, 2013; Kelley et al, 2007; Sadler et al, 2007). Whether reconsolidation of more complex memories, such as those formed during cocaine self-administration, can also be disrupted with NMDAr blockade remains unclear. For example, some research suggests that habitual learning, including operant 
conditioning, does not undergo reconsolidation (Brown et al, 2008; Hernandez and Kelley, 2004; Mierzejewski et al, 2009), whereas other research with different parameters suggests that it does (Exton-McGuinness and Lee, 2015; ExtonMcGuinness et al, 2014; Milton et al, 2008; Wells et al, 2016; Wouda et al, 2010).

We previously found that blocking NMDArs before four 45-min extinction sessions disrupted extinction following cocaine self-administration (Hafenbreidel et al, 2014). Here, we aimed to determine if NMDArs were necessary for extinction consolidation, and in which brain region. We examined the effects of NMDAr blockade in the infralimbic region of the medial prefrontal cortex (IL-mPFC). This region is implicated in extinction consolidation of conditioned fear (Quirk and Mueller, 2008; Quirk et al, 2000; Santini et al, 2001), conditioned place preference (Otis et al, 2014), and self-administration (LaLumiere et al, 2010; Peters et al, 2008). We also targeted NMDArs in the nucleus accumbens (NAc) shell (NAc shell) as this structure receives projections from IL-mPFC (Groenewegen et al, 1999), and has been implicated in both extinction (Peters et al, 2008) and reconsolidation (Miller and Marshall, 2005). However, we found that blocking NMDArs after four 45-min extinction sessions resulted in a reduction in lever pressing, which suggested facilitated extinction or disrupted reconsolidation of the original drug-cue memory. Therefore, to determine which of these processes were being modulated by NMDAr blockade, we investigated the role of NMDArs in IL-mPFC during reconsolidation of cocaine selfadministration memory.

\section{MATERIALS AND METHODS}

\section{Subjects}

Male Long-Evans rats (Harlan) weighing 250-300 g were housed and handled as previously described (Hafenbreidel et al, 2014). Rats had unlimited water access, but were food restricted (13-28 g rat chow/day) throughout experiments except during surgery and recovery. Protocols were approved by the Institutional Animal Care and Use Committee at the University of Wisconsin-Milwaukee in accordance with National Institutes of Health guidelines.

\section{Drugs}

Cocaine $\mathrm{HCl}$ (NIDA) was dissolved in sterile $0.9 \%$ saline and administered intravenously (i.v.) at $0.25 \mathrm{mg}$ per infusion. $( \pm)$ 3-(2-carboxypiperazin-4yl)propyl-1-phosphonic acid (CPP; Tocris) was dissolved in sterile $0.9 \%$ saline and administered at a dose of $10 \mathrm{mg} / \mathrm{kg}$, i.p., or was infused into IL-mPFC $(36 \mu \mathrm{g} / 0.3 \mu \mathrm{l} / \mathrm{side}$ at a rate of $0.2 \mu \mathrm{l} / \mathrm{min}) 1 \mathrm{~h}$ before or immediately after extinction training. Ro25-6981 (Ro25; $2 \mu \mathrm{g} / \mu \mathrm{l}$, Sigma-Aldrich), NVP-AAM077 (NVP; $1 \mu \mathrm{g} / \mu \mathrm{l}$, Sigma-Aldrich), or vehicle (10\% DMSO in $\left.\mathrm{dH}_{2} \mathrm{O}\right)$ was infused into IL-mPFC or NAc shell $(0.3 \mu \mathrm{l}$ per side at a rate of $0.5 \mu \mathrm{l} / \mathrm{min}$ ) immediately following extinction or reactivation sessions. These doses were determined based on previous research (Burgos-Robles et al, 2007; Gilmartin et al, 2013; Hafenbreidel et al, 2014; Santini et al, 2001).

\section{Self-administration}

Self-administration procedures were conducted using 12 sound-attenuated operant conditioning chambers (MED Associates) as previously described (Hafenbreidel et al, 2014). Briefly, 1 week after arrival, rats were food restricted for 3 days before sucrose training sessions on a fixed ratio-1 (FR1) schedule of reinforcement. Next, rats were anesthetized with a mixture of ketamine/xylazine $(87 / 13 \mathrm{mg} / \mathrm{kg}$, i.p.) and implanted with chronic intravenous catheters (assembled by Access Technologies) as described previously (Hafenbreidel et al, 2014). Rats in infusion experiments were implanted with a 26-gauge double-barrel guide cannula aimed bilaterally at IL-mPFC (AP, +2.8, ML, $\pm 0.6, \mathrm{DV}$, $-4.4 \mathrm{~mm}$ relative to bregma) or NAc shell (AP, $+1.5, \mathrm{ML}$, $\pm 0.6, \mathrm{DV},-6 \mathrm{~mm}$ relative to bregma; see Otis et al, 2013 for cannula surgery details). Following surgery, rats were administered penicillin $\mathrm{g}$ procaine $(75,000$ units $/ 0.35 \mathrm{ml}$, s.c.) and carprofen $(5 \mathrm{mg} / 0.1 \mathrm{ml}$, s.c.). Following recovery, catheter patency was verified with $0.3 \mathrm{ml}$ of $1 \%$ Propofol (i.v.), a short acting anesthetic that causes immediate loss of muscle tone. Rats were given a minimum of 7 days to recover before behavioral testing. Catheter patency was maintained daily with heparinized saline $\left(\sim 0.2 \mathrm{~cm}^{3}\right.$ of $\left.60 \mathrm{i} . \mathrm{u} . / \mathrm{ml}\right)$.

Rats were trained to self-administer cocaine daily during 90-min sessions on a FR1 schedule or until the progressive daily cap was achieved (20-35 infusions/session) for 15-25 sessions, as previously described (Hafenbreidel et al, 2014). Briefly, each session began with the house light and right stimulus light turning on, and the right and left lever extending. Responding on the active (right) lever resulted in initiation of an infusion of cocaine, onset of a tone, and offset of the stimulus light for a $20 \mathrm{~s}$ timeout period during which no lever presses were reinforced. All lever presses, reinforced or not, are reported as active lever presses during acquisition, extinction, and reinstatement. Responding on the inactive lever was recorded, but had no programmed consequences. Groups were matched on average number of infusions received during self-administration, the average number of infusions and total active and inactive lever presses made over the past 3 days of self-administration, and if rats received priming infusions or extra sessions to achieve stable drug seeking $(n=58)$.

\section{Experimental Manipulations}

Experiment one. To determine the role of NMDArs during extinction consolidation, NMDArs were blocked systemically following four 45-min extinction sessions and retention was tested on the following drug-free extinction session. Following cocaine self-administration, rats were matched and assigned to two groups receiving saline or CPP injections. Forty-five-min extinction sessions were used as previously described (Hafenbreidel et al, 2014; Hafenbreidel et al, 2015; LaLumiere et al, 2010) to minimize extinction during the first four extinction sessions, in order to better manipulate initial learning by limiting the amount of time and number of cue presentations available. Retention of the initial extinction learning was then tested during a full 90-min extinction session, after a 2-day drug wash-out period, followed by subsequent 90 -min sessions to measure extinction across days. During the first 90-min extinction session 
(extinction retention test), poor extinction memory retention from the first four 45-min sessions would be demonstrated by a significant increase in lever presses compared with controls, normal extinction retention would be demonstrated by no change in lever presses compared with controls, and good or facilitated extinction retention would be demonstrated by a significant decrease in lever presses compared with controls. During both 45- and 90-min extinction sessions, lever presses resulted in the same programmed consequences as during self-administration, but no cocaine was administered.

Experiment two. To determine the role of NMDArs in IL-mPFC during extinction of drug seeking, NMDArs were blocked before four 45-min extinction sessions and extinction retention was tested on the following 90-min drug-free extinction session. In a separate group, NMDArs were blocked immediately after four 30-min extinction sessions and extinction retention was tested on the following $90-\mathrm{min}$ drug-free extinction session to determine the role of NMDArs in IL-mPFC during the consolidation of extinction of drug seeking. Thirty-min sessions were selected for postsession infusions to further reduce extinction learning, in order to better manipulate initial extinction learning, as less time is available to acquire the new extinction memory before NMDArs are blocked during consolidation. Following cocaine self-administration, rats were matched and assigned to two groups receiving saline- or CPP-infusions into IL-mPFC. Next, rats were adapted to microinfusion procedures. On the first day, injectors extending $1.0 \mathrm{~mm}$ past the guide cannula were lowered to the infusion site for two min. The next day, saline was infused at the same rate and volume as during drug manipulation to allow the rats to adapt to changes in cranial pressure and mechanical stimulation. On the following day, saline or CPP was infused into IL-mPFC before or after the first four extinction sessions, followed by a 2-day drug wash-out break before extinction retention was tested during 90-min extinction sessions.

Experiment three. To determine the role of GluN2A- or GluN2B-containing NMDArs (GluN2A-NMDAr; GluN2BNMDAr) in IL-mPFC or NAc shell during extinction of drug seeking, GluN2A-NMDAr or GluN2B-NMDArs were blocked following four 45-min extinction sessions and retention was tested on the following drug-free extinction session. Following cocaine self-administration, rats were matched and assigned to six groups receiving vehicle, NVP, or Ro25 into IL-mPFC or NAc shell. Rats were adapted to microinfusion procedures and then infused with their respective treatment immediately following four 45-min extinction sessions. This was followed by a 2-day drug wash-out break before extinction retention was tested during 90-min extinction sessions.

Experiment four. To determine the role of GluN2ANMDARs in IL-mPFC during reconsolidation of the original drug-cue memory, GluN2A-NMDArs were blocked following four 10-min reactivation sessions (under extinction conditions) and memory retention was tested on the following drug-free extinction session. Following cocaine self-administration, rats were matched and assigned to four groups receiving vehicle or NVP into IL-mPFC immediately following four reactivation sessions, or in the absence of behavioral testing (immediately returned to their home cages with no exposure to operant chambers) for 4 days. Similarly to experiment two, rats were adapted to microinfusion procedures, infused with their respective treatments, and tested in subsequent daily $90-$ min extinction sessions.

Reinstatement procedures. Following extinction, rats received a priming injection of cocaine $(10 \mathrm{mg} / \mathrm{kg}$; i.p.) before undergoing normal extinction procedures to test for cocaineinduced reinstatement.

\section{Data Analysis}

Lever presses and infusions during acquisition were analyzed by comparing the average of the last 3 days of cocaine selfadministration between groups using $t$-tests. For extinction, lever presses were analyzed across days and between groups using a two-way repeated-measures analysis of variance (ANOVA). The last two extinction sessions for each rat were averaged and shown as the last extinction day to account for variability in the number of extinction sessions to criterion, which was defined as a significant reduction in the average active lever presses made during the last two extinction sessions compared with the first 90-min extinction session with a paired $t$-test. Reinstatement was measured by comparing the average active lever presses made during the last two extinction sessions to those made after a priming injection of cocaine, and was analyzed separately with twoway repeated measures ANOVAs. All post hoc tests were conducted, when appropriate, using Fisher's least significant difference (LSD) test. Some rats were removed from the analysis due to blocked or non-patent catheters $(n=11)$, not acquiring self-administration $(n=5)$, being two or more standard deviations away from the group mean throughout extinction $(n=1)$, or incorrect injector tip placement $(n=3)$. Final group sizes are reported in Table 1 and Figures. After behavioral procedures, verification of injector tip location was performed on cresyl violet-stained coronal sections.

\section{RESULTS}

\section{Blocking NMDArs Systemically or in IL-mPFC Does not Disrupt Extinction}

We previously found that blocking NMDArs with the NMDAr antagonist CPP before four 45-min extinction sessions disrupted extinction (Hafenbreidel et al, 2014). To determine the necessity of NMDArs during extinction consolidation following cocaine self-administration, rats were injected with saline or CPP immediately following four 45-min extinction sessions. Extinction retention was tested on days 5-23 with 90-min drug-free extinction sessions. Groups were matched by the average number of infusions, and active or inactive lever presses made during the last 3 days of cocaine self-administration (Table 1), and thus rats were anticipated to lever press similarly during extinction day 1. Both groups reduced lever pressing across the first 4 days of extinction (Figure 1a, left), but CPP-treated rats had reduced lever presses compared with saline-treated rats during the 45- and 90-min drug-free extinction sessions 
Table I Average Number of Active and Inactive Lever Presses Made or Infusions Received during the Last 3 Days of Cocaine Self-administration

\begin{tabular}{|c|c|c|c|c|c|}
\hline Experiment & Treatment & Ext days $1-4$ time & Active lever & Inactive lever & Infusions \\
\hline \multirow[t]{2}{*}{ I } & Post Sal, i.p. (6) & 45 & $47.39 \pm 12.74$ & $0.78 \pm 0.46$ & $30.00 \pm 1.72$ \\
\hline & Post CPP, i.p. (7) & 45 & $41.95 \pm 7.51$ & $1.95 \pm 1.73$ & $28.67 \pm 2.22$ \\
\hline \multirow[t]{3}{*}{2} & Pre Sal, IL (I0) & 45 & $34.70 \pm 3.42$ & $1.97 \pm 1.24$ & $28.20 \pm 1.55$ \\
\hline & Pre CPP, IL (I I) & 45 & $35.88 \pm 4.09$ & $1.15 \pm 0.52$ & $27.55 \pm 1.77$ \\
\hline & Post Sal, IL (I I) & 30 & $32.76 \pm 2.13$ & $39.58 \pm 35.25$ & $27.45 \pm 1.19$ \\
\hline & Post Ro25, IL (I0) & 45 & $35.40 \pm 5.47$ & $6.37 \pm 4.97$ & $25.43 \pm 1.79$ \\
\hline & Post NVP, IL (I0) & 45 & $37.80 \pm 10.25$ & $45.57 \pm 42.24$ & $24.97 \pm 1.83$ \\
\hline & Post Veh, NAc (I0) & 45 & $31.23 \pm 4.01$ & $2.00 \pm 1.24$ & $26.03 \pm 1.75$ \\
\hline & Post Ro25, NAc (10) & 45 & $39.20 \pm 8.24$ & $1.53 \pm 0.50$ & $26.90 \pm 1.48$ \\
\hline & NVP, IL (II) & - & $48.09 \pm \mid 0.31$ & $2.18 \pm 1.73$ & $27.12 \pm 1.22$ \\
\hline
\end{tabular}

Numbers in the parentheses are group sizes.

(Figure 1a, right). Across the 45-min extinction sessions (1-4; active lever presses), ANOVA revealed a significant effect of day $\left(F_{3,44}=3.726, p=0.018\right)$ and treatment $\left(F_{1,44}=6.241, p=0.016\right)$, but no day by treatment interaction. For the 90-min extinction sessions (5-23; days 11-22 not shown; active lever presses), ANOVA revealed a significant effect of treatment $\left(F_{1,209}=56.044, p<0.0001\right)$ but no effect of day or day by treatment interaction. A summary of the statistics for inactive lever presses for all experiments are shown in Table 2, and are described in Supplementary Results (data not shown). Moreover, further analysis of extinction day 5 (day 1 for the final experiment) within session lever pressing is described in Supplementary Results for each experiment and shown in Supplementary Figures 1 and 2. Following extinction, all rats were tested for cocaineinduced reinstatement of drug seeking. Rats were given a non-contingent priming injection of cocaine (Figure 1b) before a 90-min extinction session. ANOVA revealed a significant increase in active lever pressing during the reinstatement test compared with the last extinction session $\left(F_{1,22}=22.942, p<0.0001\right)$, but no effect of treatment or day by treatment interaction. Overall, these results are the opposite of our previous findings, and indicate that CPP treatment reduced active lever presses during 45- and 90-min extinction sessions, suggesting facilitated extinction or disrupted reconsolidation of the original drug-cue memory. However, both groups reinstated following a priming injection of cocaine, which suggests that the effects observed were either transient or partial.

Next, to determine if our conflicting results were due to brain penetration issues, CPP was infused directly into the brain. NMDArs in IL-mPFC have been implicated in extinction in other paradigms (eg, Otis et al, 2014; SotresBayon et al, 2009), prompting us to examine their role in extinction of cocaine seeking following self-administration. Rats were infused with saline or CPP before four 45-min extinction sessions (infusion sites depicted in Figure 1c), and retention was tested on extinction days 5-21 (90 min). Lever presses and number of infusions were equivalent between groups across the average of the last 3 days of cocaine selfadministration (Table 1). Overall, little extinction was observed in the saline-infused rats, but increased seeking was observed in the CPP-infused rats, across the first four 45-min extinction sessions (Figure 1d, left). However, CPPinfused rats had reduced lever presses compared with salineinfused rats during 90-min drug-free extinction sessions (Figure 1d, right). Across 45-min extinction sessions (1-4; active lever presses), ANOVA revealed a significant effect of treatment $\left(F_{1,76}=13.611, p<0.0001\right)$ and a day by treatment interaction $\left(F_{3,76}=3.876, p=0.012\right)$, but no effect of day. Post hoc analysis revealed that CPP-infused rats lever pressed significantly less than saline-infused rats on extinction day 1 $(p=0.047)$, but pressed more than saline-infused rats on days $2(p=0.022), 3(p=0.026)$, and $4(p=0.014)$. The increase in lever pressing in CPP-infused rats during extinction days 2-4 was likely a state-dependent effect (see Supplementary Results for locomotion results; data not shown). For the 90-min extinction sessions (5-21;11-20 not shown; active lever presses), ANOVA revealed a significant effect of day $\left(F_{16,323}=7.792, p<0.0001\right)$ and day by treatment interaction $\left(F_{16,323}=3.421, p<0.0001\right)$, but no effect of treatment. Post hoc analysis revealed that CPPinfused rats lever pressed significantly less than salineinfused rats on extinction day $5(p=0.001)$ and $6(p=0.023)$. Following extinction, all rats were tested for cocaine-induced reinstatement (Figure 1e). ANOVA revealed a significant increase in active lever pressing during the reinstatement test compared with the last extinction session $\left(F_{1,38}=54.410\right.$, $p<0.0001)$, as well as a significant effect of treatment $\left(F_{1,38}=5.389, p=0.026\right)$ and day by treatment interaction $\left(F_{1,38}=4.953, p=0.032\right)$. Post hoc analysis revealed that previously CPP-treated rats lever pressed more than 
C

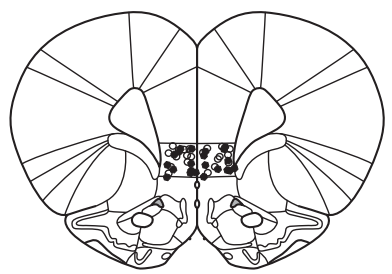

f

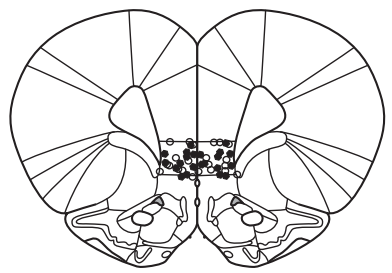

a

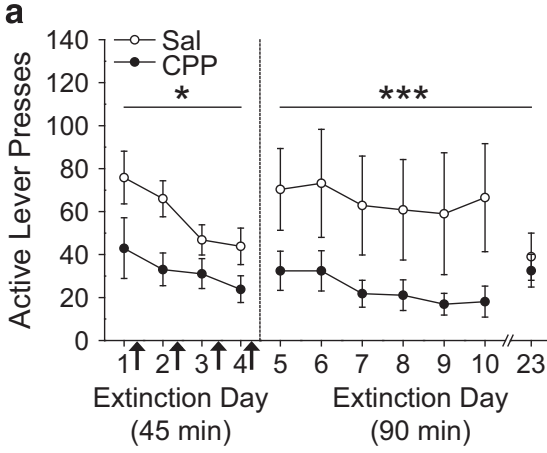

d

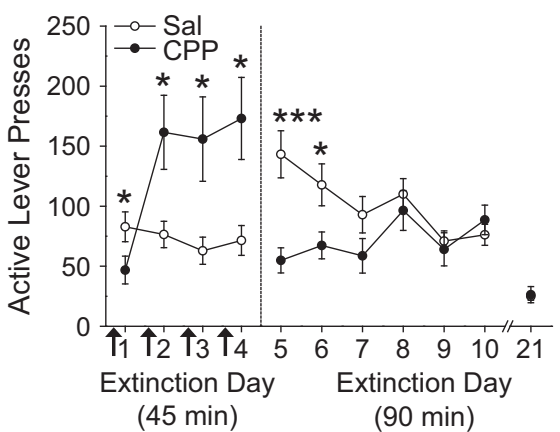

g

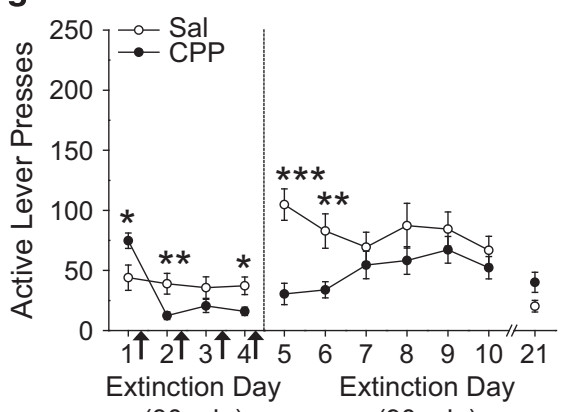

b

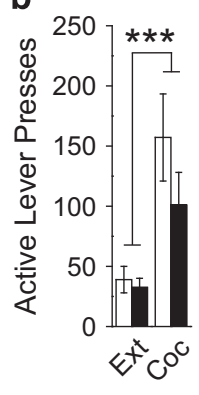

e

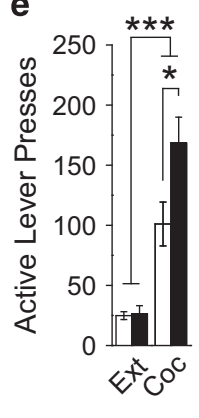

h

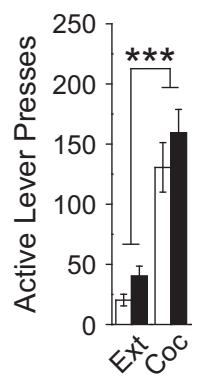

(30 min)

(90 $\mathrm{min})$

Figure I Blocking NMDArs systemically or in IL-mPFC does not disrupt extinction consolidation, but rather, reduces subsequent lever pressing. (a) Postextinction injections of CPP (arrows) decreased lever pressing during extinction, specifically during the 90-min extinction session when retention was tested. Sal, $n=6$; CPP, $n=7$. (b) All rats reinstated following a priming injection of cocaine. (c) Injector tip locations for saline (open circles; $n=10$ ) or CPP (closed octagons; $n=11$ ) in IL-mPFC with pre-extinction infusions. (d) Pre-extinction infusions of CPP (arrows) into IL-mPFC increased lever pressing during extinction days 2-4, but resulted in reduced lever pressing during the 90 -min extinction sessions when retention was tested. (e) All rats reinstated following a priming injection of cocaine, but previously CPP-treated rats were potentiated. (f) Injector tip locations for saline (open circles; $n=1 \mathrm{I}$ ) or CPP (closed octagons; $n=1 \mathrm{I}$ ) in IL-mPFC with post-extinction infusions. (g) Post-extinction infusions of CPP (arrows) in IL-mPFC reduced lever pressing on extinction days 2 and 4, and during the 90-min extinction sessions when retention was tested. (h) All rats reinstated following a priming injection of cocaine. Error bars are \pm SEM. $* p<0.05, * * p<0.01, * * * * 0.0001$.

previously saline-treated rats following a priming injection of cocaine $(p=0.029)$. Overall, blocking infralimbic NMDArs one $\mathrm{h}$ before four 45 -min extinction sessions resulted in similar effects as post-session systemic CPP administration during extinction day 5 , as rats lever pressed less than salinetreated rats. Moreover, both groups reinstated following a priming injection of cocaine, but previously CPP-treated rats had potentiated seeking, which may be due to nonspecific drug effects of CPP (Del Arco et al, 2011; Del Arco et al, 2008) being active during memory retrieval.

To dissociate acquisition from consolidation of extinction, we examined the role of NMDArs in IL-mPFC following extinction of cocaine seeking. Rats were infused with saline or CPP immediately following four 30-min extinction sessions (infusion sites depicted in Figure 1f), and retention was tested on extinction days 5-21 (90 min). Lever presses and number of infusions were equivalent between groups across the average of the last 3 days of cocaine selfadministration (Table 1). Both treatment groups reduced lever pressing across the first 4 days of extinction (Figure 1g, left), but CPP-infused rats had reduced lever presses compared with saline-infused rats during the 90-min drugfree extinction sessions (Figure 1g, right). Across the 30-min extinction sessions ( $1-4$; active lever presses), ANOVA revealed a significant effect of day $\left(F_{3,80}=10.338, p<0.0001\right)$ and day by treatment interaction $\left(F_{3,80}=6.706, p<0.0001\right)$, 
Table 2 Statistical Summary of Inactive Lever Presses Made During Extinction and Cocaine-Induced Reinstatement

\begin{tabular}{|c|c|c|c|c|c|}
\hline \multirow[t]{3}{*}{ I } & Post Sal, i.p. & 45 & $\operatorname{Day}^{\mathrm{NS}}$ & Day*** & Day*** \\
\hline & Post CPP, i.p. & 45 & Treat粎米 & Treat米米 & Treat* \\
\hline & & & DayXTreat ${ }^{N S}$ & DayXTreat* & DayXTreat ${ }^{N S}$ \\
\hline \multirow[t]{4}{*}{2} & Pre Sal, IL & 45 & NS & NS & NS \\
\hline & Pre CPP, IL & 45 & & & \\
\hline & Post CPP, IL & 30 & Treat NS & & \\
\hline & & & DayXTreat $^{\text {NS }}$ & & \\
\hline \multirow[t]{8}{*}{3} & Post Veh & 45 & Day $^{N S}$ & NS & NS \\
\hline & Post NVP, IL & 45 & Treat* & & \\
\hline & & & DayXTreat ${ }^{N S}$ & & \\
\hline & Post NVP, NAc & 45 & Treat* & Treat****** & \\
\hline & & & DayXTreat ${ }^{\text {NS }}$ & DayXTreat ${ }^{N S}$ & \\
\hline & Post Veh & 45 & Day* & NS & NS \\
\hline & Post Ro25, NAc & 45 & Treat $^{\text {NS }}$ & & \\
\hline & & & DayX Treat $^{\text {NS }}$ & & \\
\hline \multirow[t]{5}{*}{4} & Post Veh, IL & 10 & NS & Day***** & Day* \\
\hline & Post NVP, IL & 10 & & Treat $^{\text {NS }}$ & Treat $^{N S}$ \\
\hline & & & & DayXTreat ${ }^{N S}$ & DayXTreat ${ }^{N S}$ \\
\hline & Veh, IL & - & - & Day**** & Day*** \\
\hline & NVP, IL & - & & Treat*** & Treat $^{\text {NS }}$ \\
\hline
\end{tabular}

Abbreviation: NS, not significant.

* $p<0.05$; $* * * 0.01$; **** $p<0.0001$.

but no effect of treatment. Post hoc analysis revealed that CPP-infused rats lever pressed significantly more than saline-infused rats on extinction day $1(p=0.021)$, but significantly less on extinction days $2(p=0.009)$, and 4 $(p=0.016)$. The significant increase in lever pressing by CPP-infused rats on extinction day 1 was not expected as rats did not significantly differ on average number of infusions, active or inactive lever presses made during the last 3 days of acquisition (Table 1), and CPP was infused following the extinction session. For 90-min extinction days $(5-21 ; 11-20$ not shown; active lever presses), ANOVA revealed a significant effect of day $\left(F_{16,340}=3.361, p<0.0001\right)$ and day by treatment interaction $\left(F_{16,340}=3.795, p<0.0001\right)$, but no effect of treatment. Post hoc analysis revealed that CPPinfused rats lever pressed significantly less than salineinfused rats on extinction days $5(p<0.0001), 6(p=0.006)$, but significantly more on day 20 ( $p=0.049$; data not shown). The significant increase in responding on extinction day 20 could be due to spontaneous recovery in the CPP-infused rats, which would suggest that the effect observed on days 5 and 6 was transient or partial. Following extinction, all rats were tested for cocaine-induced reinstatement (Figure 1h). ANOVA revealed a significant increase in active lever pressing during the reinstatement test compared with the last extinction session $\left(F_{1,38}=58.104, p<0.0001\right)$, but no effect of treatment or day by treatment interaction. Overall, blocking infralimbic NMDArs immediately following four 30-min extinction sessions did not impair extinction, but instead resulted in effects similar to post-session systemic CPP administration. Moreover, similar to post-session systemic injections of CPP, both groups reinstated following a priming injection of cocaine similarly to saline-treated rats, which suggests that post-session administration of CPP (systemic or into IL-mPFC) induces a transient or partial reduction in lever pressing during the 90-min extinction sessions.

\section{In IL-mPFC and NAc Shell, Blocking GluN2A-NMDArs Does not Disrupt Extinction}

CPP has a higher affinity for GluN2A-NMDArs than to GluN2B-NMDARs (Lehmann et al, 1986; Lozovaya et al, 2004) and has some nonspecific effects (Del Arco et al, 2011; Del Arco et al, 2008), which could underlie our conflicting results. Therefore, to control for drug-specific effects and to dissociate the role of NMDAr subtypes in IL-mPFC and its downstream target the NAc shell during extinction, we first isolated the effects of GluN2A-NMDAr inhibition following extinction. Rats were infused with vehicle or NVP 
a

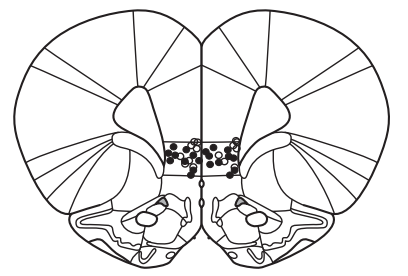

d

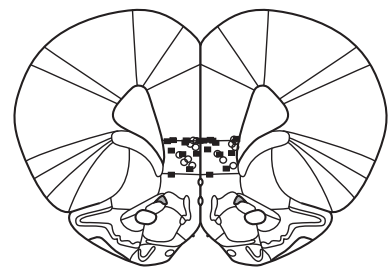

g

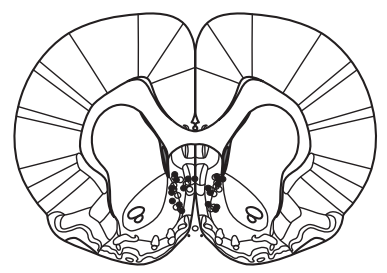

j

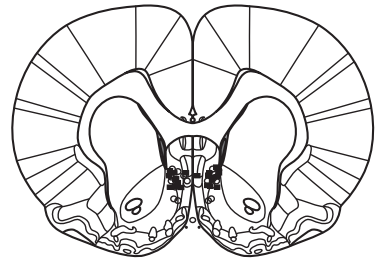

b

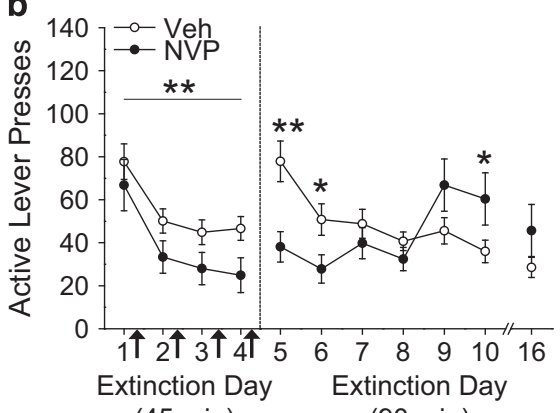

(45 min)

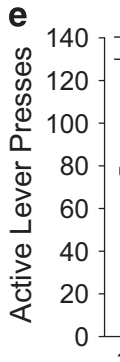

${ }_{1}^{1} 2 \uparrow 3 \uparrow 4 \uparrow$

(45 min)

h

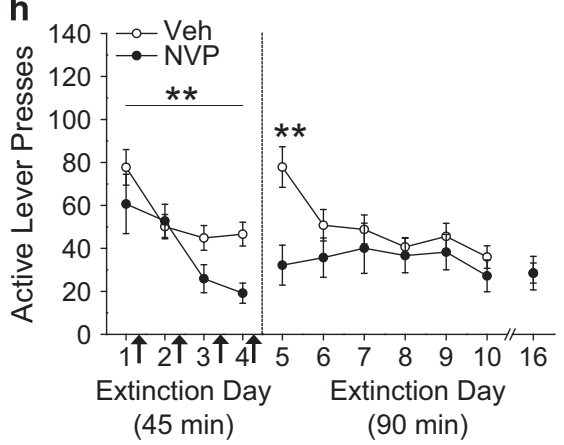

k

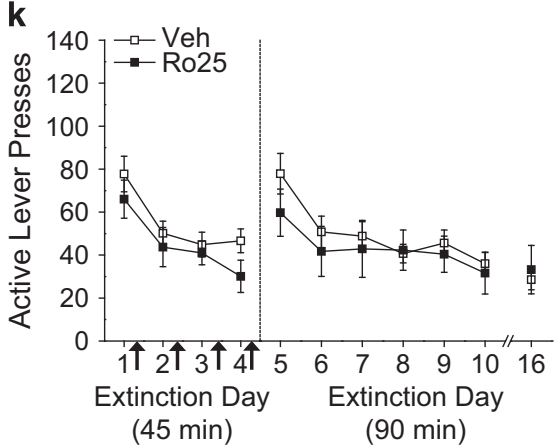

C
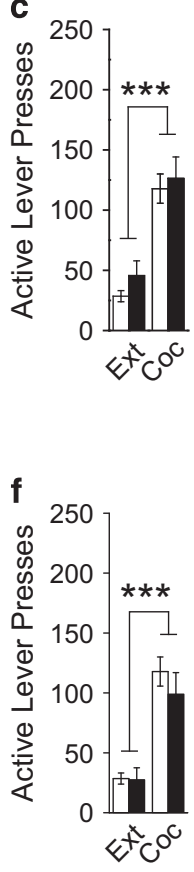
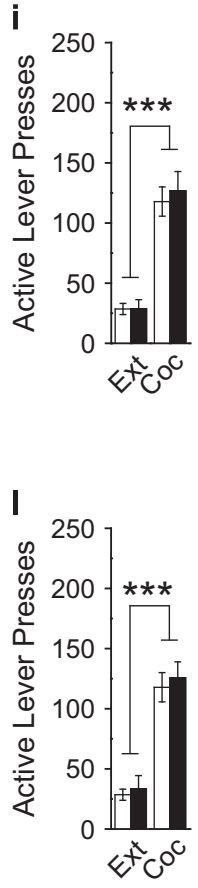

Figure 2 In IL-mPFC or NAc shell, blocking GluN2A-containing NMDArs does not disrupt extinction, but rather, reduces subsequent lever pressing. (a) Injector tip locations for vehicle (open circles; $n=9$ ) or NVP (closed circles; $n=10$ ) in IL-mPFC. (b) Post-extinction infusions of GluN2A-specific NVP (arrows) into IL-mPFC resulted in reduced lever pressing during extinction, specifically during the 90 -min extinction sessions when retention was tested. (c) All rats reinstated following a priming injection of cocaine. (d) Injector tip locations for vehicle (open circles; $n=9$ ) or Ro25 (closed squares; $n=10$ ) in IL-mPFC. (e) Post-extinction infusions of GluN2B-specific Ro25 (arrows) into IL-mPFC had no effect on extinction. (f) All rats reinstated following a priming injection of cocaine. (g) Injector tip locations for vehicle (open circles; $n=10$ ) or NVP (closed circles; $n=10$ ) in NAc shell. (h) Post-extinction infusions of GluN2A-specific NVP (arrows) into NAc shell resulted in reduced lever pressing during extinction, specifically during the 90-min extinction sessions when retention was tested. (i) All rats reinstated following a priming injection of cocaine. (j) Injector tip locations for vehicle (open circles; $n=10$ ) or Ro25 (closed squares; $n=10$ ) in $N A c$ shell. (k) Post-extinction infusions of GluN2B-specific Ro25 (arrows) into NAc shell had no effect on extinction. (I) All rats reinstated following a priming injection of cocaine. Error bars are \pm SEM. $* p<0.05$, *** $<0.0$ I, **** $p<0.000$ I. 
immediately following four 45-min extinction sessions (infusion sites depicted in Figure 2a), and retention was tested on extinction days 5-16 (90 min). Lever presses and number of infusions were equivalent between groups across the average of the last 3 days of cocaine self-administration (Table 1). Vehicle-infused rats in IL-mPFC or NAc shell were not significantly different during acquisition (Table 1; active or inactive, all $t s<1.264, p s>0.05$ ), extinction (active or inactive, all $F s<0.600, p s>0.05$ ), or reinstatement (active or inactive, all $F s<0.469$, $p s>0.05$ ), and therefore were collapsed into a single vehicle group. Both vehicle and NVPinfused groups reduced lever pressing across the first 4 days of extinction (Figure 2b, left), but NVP-infused rats had reduced lever presses compared with vehicle-infused rats during the 90-min drug-free extinction sessions (Figure 2b, right). Across the 45-min extinction sessions (1-4; active lever presses), ANOVA revealed a significant effect of day $\left(F_{3,108}=10.037, p<0.0001\right)$ and treatment $\left(F_{1,108}=9.155\right.$, $p=0.003)$, but no day by treatment interaction. For the 90-min extinction sessions (5-16; 11-15 not shown; active lever presses), ANOVA revealed a significant effect of day $\left(F_{11,324}=2.362, p=0.008\right)$ and day by treatment interaction $\left(F_{11,324}=3.006, p=0.001\right)$, but no effect of treatment. Post hoc analysis revealed that NVP-infused rats lever pressed significantly less than saline-infused rats on extinction days 5 $(p=0.009)$ and $6(p=0.049)$, but pressed significantly more than saline-infused rats on extinction day $10(p=0.040)$. The significant increase in responding on extinction day 10 could be due to spontaneous recovery in the NVP-infused rats, which would suggest that the effect observed on days 5 and 6 was transient or partial. Following extinction, all rats were tested for cocaine-induced reinstatement (Figure 3c). ANOVA revealed a significant increase in active lever pressing during the cocaine-induced reinstatement test compared with the last extinction session $\left(F_{1,54}=51.211, p<0.0001\right)$, but no effect of treatment or day by treatment interaction. Overall, blocking infralimbic GluN2A-NMDArs immediately following four 45-min extinction sessions produced results similar to infusions of the nonspecific NMDAr antagonist CPP into IL-mPFC during the 90-min extinction sessions. These results suggest that the reduction in lever pressing observed during the extinction retention tests (eg, extinction day 5) were due to GluN2A-NMDArs blockade selectively and not likely due to a drug-specific effect. In addition, both groups reinstated following a priming injection of cocaine, which suggests that post-session blockade of GluN2ANMDARs induces transient or partial reduction in lever pressing during the 90-min extinction sessions.

Next, we examined the role of GluN2B-NMDArs in IL-mPFC during extinction by infusing rats with vehicle or Ro25 immediately following four 45-min extinction sessions (infusion sites depicted in Figure 2d), and testing retention a

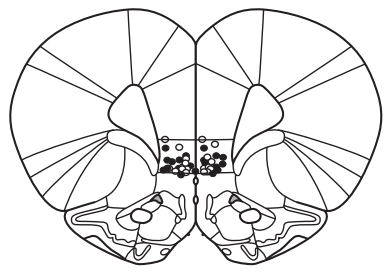

d

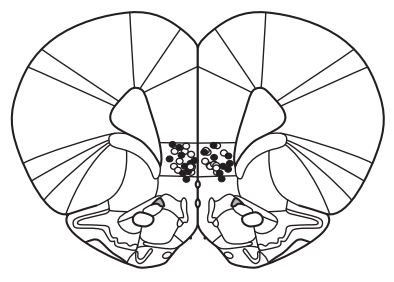

b

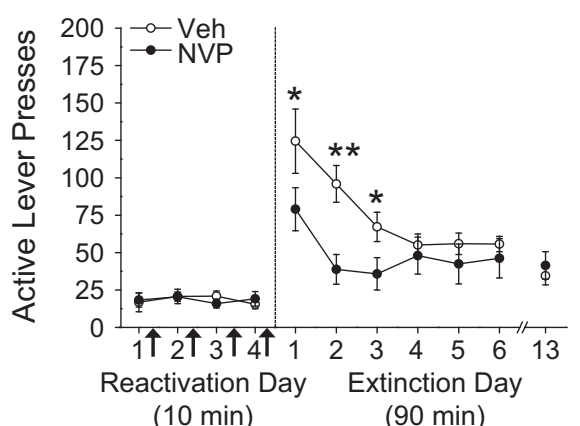

e

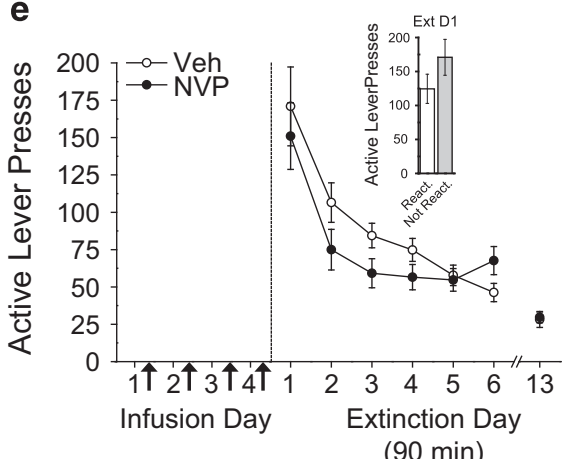

C

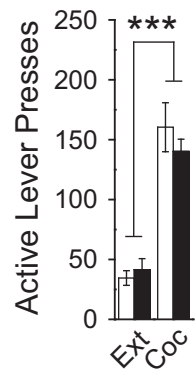

f

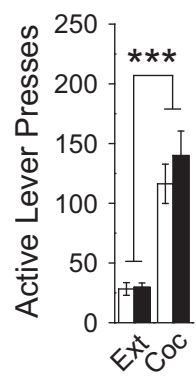

Figure 3 Blocking GluN2A-containing NMDArs in IL-mPFC following four 10 min reactivation sessions reduced subsequent lever pressing. (a) Injector tip locations for vehicle (open circles; $n=10$ ) or NVP (closed circles; $n=10$ ) in IL-mPFC with memory reactivation. (b) Post I0-min reactivation session infusions of GluN2A-specific NVP (arrows) in IL-mPFC resulted in reduced lever pressing during the 90-min extinction sessions when retention was tested. (c) All rats reinstated following a priming injection of cocaine. (d) Injector tip locations for vehicle (open circles; $n=10$ ) or NVP (closed circles; $n=1 \mathrm{I}$ ) in IL-mPFC without memory reactivation. (e) Infusions of NVP into IL-mPFC without memory reactivation had no effect on lever pressing during the 90 -min extinction sessions when retention was tested. (e, inset) Vehicle-treated rats that did undergo the 10-min reactivation sessions lever pressed similarly to vehicle-treated rats that did not undergo the I0-min reactivation sessions during extinction day I. (f) All rats reinstated following a priming injection of cocaine. Error bars are \pm SEM. $* p<0.05, * * p<0.01, * * * * 0<0.0001$. 
during extinction days 5-16 (90 min). Lever presses and number of infusions were equivalent between groups across the average of the last 3 days of cocaine self-administration (Table 1). Both treatment groups lever pressed similarly throughout extinction (Figure 2e). Across the 45-min extinction sessions ( $1-4$; active lever presses), ANOVA revealed a significant effect of day $\left(F_{3,108}=2.741, p=0.047\right)$, but no effect of treatment or day by treatment interaction. For the 90-min extinction sessions (5-16;11-15 not shown; active lever presses), ANOVA revealed a significant effect of day $\left(F_{11,324}=9.101, p<0.0001\right)$, but no effect of treatment or a day by treatment interaction. Following extinction, all rats were tested for cocaine-induced reinstatement (Figure 2f). ANOVA revealed a significant increase in active lever pressing during the cocaine-induced reinstatement test compared with the last extinction session $\left(F_{1,54}=47.581, p<0.0001\right)$, but no effect of treatment or day by treatment interaction. Thus, blocking infralimbic GluN2B-NMDArs following four 45-min extinction sessions had no effect on extinction or cocaine-induced reinstatement.

Furthermore, to determine if our results were specific to infralimbic GluN2A-NMDArs, rats were infused with vehicle or NVP into NAc shell immediately following four 45-min extinction sessions (infusion sites depicted in Figure $2 \mathrm{~g}$ ), and retention was tested during extinction days 5-16 (90 $\mathrm{min}$ ). Lever presses and number of infusions were equivalent between groups across the average of the last 3 days of cocaine self-administration (Table 1). Both treatment groups reduce lever pressing across the first 4 days of extinction (Figure 2e, left), but NVP-infused rats had reduced lever presses compared with vehicle-infused rats during the 90min drug-free extinction sessions (Figure 2e, right). Across the 45-min extinction sessions (1-4; active lever presses), ANOVA revealed a significant effect of day $\left(F_{3,108}=9.485\right.$, $p<0.0001)$ and treatment $\left(F_{1,108}=7.761, p=0.006\right)$, but no day by treatment interaction. For the 90 -min extinction days (5-16;11-15 not shown; active lever presses), ANOVA revealed a significant effect of day $\left(F_{11,324}=3.191\right.$, $p<0.0001)$, treatment $\left(F_{1,324}=6.426, p=0.012\right)$, and day by treatment interaction $\left(F_{11,324}=1.894, p=0.039\right)$. Post hoc analysis revealed that NVP-infused rats lever pressed significantly less than saline-infused rats on extinction day $5(p=0.004)$. Following extinction, all rats were tested for cocaine-induced reinstatement (Figure 2i). ANOVA revealed a significant increase in active lever pressing during the cocaine-induced reinstatement test compared with the last extinction session $\left(F_{1,54}=71.671, p<0.0001\right)$, but no effect of treatment or day by treatment interaction. Overall, blocking GluN2A-NMDArs in NAc shell immediately following four 45-min extinction sessions resulted in reduced lever pressing during the initial 90-min extinction sessions, but had no effect on cocaine-induced reinstatement, which are similar to the results observed following infusions into IL-mPFC. These results suggest that blocking GluN2A-containing NMDArs in the NAc shell resulted in a transient or partial reconsolidation disruption or facilitated extinction.

In addition, to determine the role of GluN2B-NMDArs in NAc shell during extinction, rats were infused with vehicle or Ro25 immediately following four 45-min extinction sessions (infusion sites depicted in Figure 2j), and retention was tested during extinction days 5-16 (90 min). Lever presses and number of infusions were equivalent between groups across the average of the last 3 days of cocaine selfadministration (Table 1). Both treatment groups lever pressed similarly throughout extinction (Figure 2k). Across the 45-min extinction sessions ( $1-4$; active lever presses), ANOVA revealed a significant effect of day $\left(F_{3,108}=8.107\right.$, $p<0.0001$ ), but no effect of treatment or day by treatment interaction. For the 90-min extinction sessions (5-16; $11-15$ not shown; active lever presses), ANOVA revealed a significant effect of day $\left(F_{11,324}=5.109, p<0.0001\right)$, but no effect of treatment or a day by treatment interaction. Following extinction, all rats were tested for cocaineinduced reinstatement (Figure 2l). ANOVA revealed a significant increase in active lever pressing during the cocaine-induced reinstatement test compared with the last extinction session $\left(F_{1,54}=68.773, p<0.0001\right)$, but no effect of treatment or day by treatment interaction. Thus, blocking GluN2B-NMDArs in NAc shell immediately following four 45-min extinction sessions had no effect on extinction or cocaine-induced reinstatement.

\section{In IL-mPFC, GluN2A-NMDArs Modulate Reconsolidation of the Original Drug-Cue Memory}

We found that post $30-$ or $45-\mathrm{min}$ extinction session systemic injections or infusions into IL-mPFC of CPP, or infusions into IL-mPFC or NAc of NVP (but not Ro25) resulted in a reduction of lever pressing when tested during a subsequent $90-$ min extinction retention test. These results suggest that NMDAr blockade results in either facilitated extinction or disrupted reconsolidation of the original drugcue memory. To distinguish which of these processes resulted in the significant reduction in lever pressing observed during the extinction retention tests, we directly tested whether infralimbic GluN2A-NMDArs are necessary for reconsolidation of the original drug memory by utilizing 10 -min reactivation sessions that are insufficient to induce extinction (see below for statistical anaylsis and Figure $3 \mathrm{e}$ inset; see also Fuchs et al, 2009; Nader et al, 2000; Tronson and Taylor, 2007). Following cocaine self-administration, rats were infused with vehicle or NVP immediately following four 10-min reactivation sessions (infusion sites depicted in Figure 3a). Memory retention was then tested during subsequent 90 -min extinction sessions. Lever presses and number of infusions were equivalent between groups across the average of the last 3 days of cocaine self-administration (Table 1). Both treatment groups lever pressed similarly during the four 10-min reactivation sessions (Figure 3b, left), but NVP-infused rats had reduced lever presses compared with vehicle-infused rats during the 90-min drug-free extinction sessions (Figure 3b, right). Across the 10-min reactivation sessions (1-4; active lever presses), ANOVA did not reveal a significant effect of day, treatment, or a day by treatment interaction. For the 90-min extinction days (1-13; 7-12 not shown; active lever presses), ANOVA revealed a significant effect of day $\left(F_{12,234}=10.148, p<0.0001\right)$, treatment $\left(F_{1,234}=8.510, p=0.004\right)$, and day by treatment interaction $\left(F_{12,234}=3.122, p<0.0001\right)$. Post hoc analysis revealed that NVP-infused rats lever pressed significantly less than saline-infused rats on extinction day $1(p=0.019), 2$ $(p=0.002)$, and $3(p=0.045)$. Following extinction, all rats were tested for cocaine-induced reinstatement (Figure $3 c$ ). 
ANOVA revealed a significant increase in active lever pressing during the cocaine-induced reinstatement test compared with the last extinction session $\left(F_{1,36}=78.525\right.$, $p<0.0001$ ), but no effect of treatment or day by treatment interaction. Thus, blocking infralimbic GluN2A-NMDArs immediately following four reactivation sessions significantly reduced lever pressing suggesting that reconsolidation of the original drug-cue memory was disrupted. However, both treatment groups reinstate following a priming injection of cocaine, which suggests a partial or transient disruption.

To determine if reactivation was required for reconsolidation disruption, we blocked infralimbic GluN2A-NMDArs in the absence of reactivation sessions. Rats were infused with vehicle or NVP for 4 days and immediately returned to their home cages without behavioral testing (infusion sites depicted in Figure 3d). Memory retention was then tested during subsequent 90-min extinction sessions. Lever presses and number of infusions were equivalent between groups across the average of the last 3 days of cocaine selfadministration (Table 1). Rats were infused with vehicle or NVP into IL-mPFC for 4 days and immediately returned to their home cages without behavioral testing (Figure $3 \mathrm{e}$, left). Treatment groups did not differ during the 90-min drug-free extinction sessions (Figure 3e, right; days 1-13; 7-12 not shown; active lever presses), as ANOVA revealed a significant effect of day $\left(F_{12,247}=21.535, p<0.0001\right)$, but no effect of treatment or a day by treatment interaction. In addition, active lever presses made during extinction day 1 by vehicle-infused rats that did undergo four 10-min reactivation sessions were not different than vehicle-infused rats that did not undergo four 10-min reactivation sessions $(t(18)=-1.121, p=0.277$; Figure $3 \mathrm{e}$ inset). Thus, four 10 -min reactivation sessions were not sufficient to induce extinction, as evident by a lack of a significant reduction in lever pressing, as shown by others (Fuchs et al, 2009; Nader et al, 2000; Tronson and Taylor, 2007). Following extinction, all rats were tested for cocaine-induced reinstatement (Figure 3f). ANOVA revealed a significant increase in active lever pressing during the cocaine-induced reinstatement test compared with the last extinction session $\left(F_{1,38}=52.436\right.$, $p<0.0001$ ), but no effect of treatment or day by treatment interaction. Overall, blocking GluN2A-NMDArs in IL-mPFC following memory reactivation transiently or partially disrupted reconsolidation of the original drug-cue memory, but blocking GluN2A-NMDArs without memory reactivation had no effect on subsequent behavior. However, blocking GluN2A-NMDArs in IL-mPFC with or without memory reactivation did not alter cocaine-induced reinstatement.

\section{DISCUSSION}

Here, we aimed to determine the role of NMDArs during extinction consolidation following self-administration. Instead, we found that inhibiting NMDArs, specifically the GluN2A-NMDAr, systemically or in IL-mPFC or NAc shell resulted in reduced lever pressing during the extinction retention test (extinction day 5 ). These results suggested that blocking NMDArs after four 45- or 30-min extinction sessions either enhanced extinction consolidation or disrupted the reconsolidation of the original drug-cue memory.
We directly tested these alternatives by blocking infralimbic GluN2A-NMDArs following four 10-min reactivation sessions, which resulted in a reduction in lever pressing during the retention test (extinction day 1) in NVP-infused rats compared with vehicle-infused rats. However, in each experiment, rats treated with the NMDAr antagonist reinstated lever pressing following a priming injection of cocaine. Therefore, we conclude that the effect of GluN2ANMDAr blockade observed during the 90-min retention sessions was likely due to partial or transient reconsolidation disruption rather than facilitated extinction.

To date, the role of GluN2A-NMDArs in IL-mPFC during reconsolidation or extinction following cocaine selfadministration had not been investigated. Our results were surprising, as previous research has demonstrated that blocking NMDArs bidirectionally mediates learning. For example, blocking NMDArs disrupts extinction (eg, Hafenbreidel et al, 2014; Hsu and Packard, 2008; Liu et al, 2009; Santini et al, 2001) and enhancing NMDAr function facilitates extinction in a number of paradigms (Botreau et al, 2006; Fiorenza et al, 2012; Hammond et al, 2012; Langton and Richardson, 2008; Ledgerwood et al, 2003; Matsuda et al, 2010; Walker et al, 2002), including with methods used here (Hafenbreidel et al, 2014). Therefore, it seemed improbable that blocking and enhancing NMDArs would both facilitate extinction. However, under different parameters, NMDAr blockade can disrupt reconsolidation (Alaghband and Marshall, 2013; Kelley et al, 2007; Lee and Everitt, 2008; Lee et al, 2006; Pedreira et al, 2002; Sadler et al, 2007; TorrasGarcia et al, 2005), and it is more probable that NMDAr blockade would disrupt new learning and memory reconsolidation, than disrupting and facilitating new learning. Nevertheless, it is possible that NMDAr blockade with these specific parameters was facilitating extinction. Therefore, we directly tested if infralimbic GluN2A-NMDARs were necessary for reconsolidation, and concluded that infralimbic GluN2A-NMDArs were partially or transiently modulating the reconsolidation of the original drug-cue memory.

Until now, IL-mPFC did not appear to have a role in reconsolidation, as it does not mediate contextual fear reconsolidation (Mamiya et al, 2009; Stern et al, 2014). However, others have reported findings similar to ours during extinction of conditioned fear (Kwapis et al, 2014; Leaderbrand et al, 2014; Song et al, 2015). One possibility is that the mechanisms underlying reconsolidation of instrumental or complex memories may differ from classical conditioning memories due to variables such as habituation (eg, Exton-McGuinness and Lee, 2015; Hernandez and Kelley, 2004; Wells et al, 2016; Wouda et al, 2010). However, NAc has been implicated in both extinction (Peters et al, 2008) and reconsolidation (Miller and Marshall, 2005), receives projections from $\mathrm{mPFC}$ (Groenewegen et al, 1999), and is thought to underlie expression of drug seeking (Millan et al, 2011). Thus, this interaction between IL-mPFC and NAc may be critical for reconsolidation of cocaine selfadministration memory.

Our results could be explained by the different roles of NMDAr subunits in learning and memory. NMDArs are composed of four subunits, including two GluN1 subunits and typically two GluN2 subunits (GluN2A-D). The NMDAr antagonist CPP is more selective for the GluN2A-NMDAr (Lehmann et al, 1986; Lozovaya et al, 2004), which led us to 
test whether our initial results (Figure 1) were due to GluN2A-NMDAr selective blockade, as GluN2A-NMDArs are necessary for memory acquisition (Bannerman et al, 2008; Brigman et al, 2008; Dalton et al, 2012; Gilmartin et al, 2013). Moreover, blocking GluN2A-NMDArs in BLA disrupts reconsolidation of conditioned fear memory, but does not affect retrieval or memory destabilization (Milton et al, 2013), and blocking GluN2A-NMDArs in the hippocampus can impair reconsolidation of contextspecific drug-associated memories following selfadministration (Wells et al, 2016). Conversely, infralimbic GluN2B-NMDArs are necessary for consolidation of extinction of conditioned fear (Dalton et al, 2012; Sotres-Bayon et $a l, 2009)$ and drug-associated conditioned place preference memories (Otis et al, 2014). Overall, specific NMDAr subunits likely mediate different aspects of learning and memory, specifically in the formation and consolidation of the initial memory and during extinction.

Overall, our data suggests that infralimbic GluN2ANMDArs modulate reconsolidation of the original drug-cue memory. However, rats reinstated drug seeking following a priming injection of cocaine, which suggests that the observed effect was partial or transient. Conversely, the priming injection of cocaine might have been too salient of a cue, because the drug itself is the primary reinforcer, and may be resistant to reconsolidation disruption. For example, in the conditioned place preference paradigm, mice will reinstate cocaine seeking following a priming injection of cocaine even with previous reconsolidation disruption (Kelley et al, 2007). Moreover, rats were tested for cocaine reinstatement 2-5 weeks following the initial memory disruption. Little research has examined the longevity of reconsolidation disruption in a drug-associated memory following self-administration, which can potentiate over time (Pickens et al, 2011). Drug-associated memories are complex and include numerous salient and vague cues, and all associated cues may not have been retrieved and thus disrupted following GluN2A-NMDAr blockade, which could underlie the transient or partial disruption observed. Alternatively, even though our final experiment controlled for initial extinction learning, it is highly unlikely but still possible that NMDAr blockade affected extinction. Future research should examine the parameters of reconsolidation disruption by infralimbic GluN2A-NMDAr blockade. Specifically, less salient drug-associated cues should be examined during reinstatement and context-induced renewal, and the duration of the disruption including susceptibility to spontaneous recovery should be determined.

In conclusion, we demonstrated that blocking NMDArs, specifically GluN2A-NMDARs, in the IL-mPFC and NAc shell before four short extinction sessions resulted in reduced lever pressing during the extinction retention test (extinction day 5). Our findings indicate that NMDAr blockade was not facilitating extinction, but rather that the reconsolidation of the original drug-cue memory was being transiently or partially disrupted. These results suggest a potential therapeutic approach, as reducing cue-reactivity, whether through facilitated extinction or disrupted reconsolidation, could reduce relapse rates.

\section{FUNDING AND DISCLOSURE}

This research was supported by R03 DA027870, R01 DA038042, and a grant from the Puerto Rico Science, Technology and Research Trust to DM, and NIH RCMI Program grant G12MD007579. The authors declare no conflict of interest.

\section{ACKNOWLEDGMENTS}

We thank Dr Robert Twining, Dr James Otis, John Schneider, Dr Marieke Gilmartin, and Jake Burkard for technical assistance.

\section{REFERENCES}

Alaghband Y, Marshall JF (2013). Common influences of noncompetitive NMDA receptor antagonists on the consolidation and reconsolidation of cocaine-cue memory. Psychopharmacology (Berl) 226: 707-719.

Bannerman DM, Niewoehner B, Lyon L, Romberg C, Schmitt WB, Taylor A et al (2008). NMDA receptor subunit NR2A is required for rapidly acquired spatial working memory but not incremental spatial reference memory. J Neurosci 28: 3623-3630.

Botreau F, Paolone G, Stewart J (2006). d-Cycloserine facilitates extinction of a cocaine-induced conditioned place preference. Behav Brain Res 172: 173-178.

Brigman JL, Feyder M, Saksida LM, Bussey TJ, Mishina M, Holmes A (2008). Impaired discrimination learning in mice lacking the NMDA receptor NR2A subunit. Learn Mem 15: 50-54.

Brown TE, Lee BR, Sorg BA (2008). The NMDA antagonist MK-801 disrupts reconsolidation of a cocaine-associated memory for conditioned place preference but not for self-administration in rats. Learn Mem 15: 857-865.

Burgos-Robles A, Vidal-Gonzalez I, Santini E, Quirk GJ (2007). Consolidation of fear extinction requires NMDA receptordependent bursting in the ventromedial prefrontal cortex. Neuron 53: 871-880.

Childress AR, McLellan AT, O'Brien CP (1986). Role of conditioning factors in the development of drug dependence. Psychiatr Clin North Am 9: 413-425.

Dalton GL, Wu DC, Wang YT, Floresco SB, Phillips AG (2012). NMDA GluN2A and GluN2B receptors play separate roles in the induction of LTP and LTD in the amygdala and in the acquisition and extinction of conditioned fear. Neuropharmacology 62: 797-806.

Del Arco A, Ronzoni G, Mora F (2011). Prefrontal stimulation of GABAA receptors counteracts the corticolimbic hyperactivity produced by NMDA antagonists in the prefrontal cortex of the rat. Psychopharmacology 214: 535-536.

Del Arco A, Segovia G, Mora F (2008). Blockade of NMDA receptors in the prefrontal cortex increases dopamine and acetylcholine release in the nucleus accumbens and motor activity. Psychopharmacology (Berl) 201: 325-338.

Exton-McGuinness MT, Lee JL (2015). Reduction in responding for sucrose and cocaine reinforcement by disruption of memory reconsolidation. eNeuro 2. doi:10.1523/ENEURO.0009-15.2105.

Exton-McGuinness MT, Patton RC, Sacco LB, Lee JL (2014). Reconsolidation of a well-learned instrumental memory. Learn Mem 21: 468-477.

Fiorenza NG, Rosa J, Izquierdo I, Myskiw JC (2012). Modulation of the extinction of two different fear-motivated tasks in three distinct brain areas. Behav Brain Res 232: 210-216.

Fuchs RA, Bell GH, Ramirez DR, Eaddy JL, Su ZI (2009). Basolateral amygdala involvement in memory reconsolidation processes that facilitate drug context-induced cocaine seeking. Eur J Neurosci 30: 889-900. 
Gilmartin MR, Kwapis JL, Helmstetter FJ (2013). NR2A- and NR2B-containing NMDA receptors in the prelimbic medial prefrontal cortex differentially mediate trace, delay, and contextual fear conditioning. Learn Mem 20: 290-294.

Groenewegen HJ, Galis-de Graaf Y, Smeets WJ (1999). Integration and segregation of limbic cortico-striatal loops at the thalamic level: an experimental tracing study in rats. J Chem Neuroanat 16: 167-185.

Hafenbreidel M, Rafa Todd C, Twining RC, Tuscher JJ, Mueller D (2014). Bidirectional effects of inhibiting or potentiating NMDA receptors on extinction after cocaine self-administration in rats. Psychopharmacology (Berl) 231: 4585-4594.

Hafenbreidel M, Twining RC, Rafa Todd C, Mueller D (2015). Blocking infralimbic basic fibroblast growth factor (bFGF or FGF2) facilitates extinction of drug seeking after cocaine selfadministration. Neuropsychopharmacology 40: 2907-2915.

Hammond S, Seymour CM, Burger A, Wagner JJ (2012). D-serine facilitates the effectiveness of extinction to reduce drug-primed reinstatement of cocaine-induced conditioned place preference. Neuropharmacology 64: 464-471.

Hernandez PJ, Kelley AE (2004). Long-term memory for instrumental responses does not undergo protein synthesis-dependent reconsolidation upon retrieval. Learn Mem 11: 748-754.

Hsu E, Packard MG (2008). Medial prefrontal cortex infusions of bupivacaine or AP-5 block extinction of amphetamine conditioned place preference. Neurobiol Learn Mem 89: 504-512.

Kelley JB, Anderson KL, Itzhak Y (2007). Long-term memory of cocaine-associated context: disruption and reinstatement. Neuroreport 18: 777-780.

Kwapis JL, Jarome TJ, Helmstetter FJ (2014). The role of the medial prefrontal cortex in trace fear extinction. Learn Mem 22: 39-46.

LaLumiere RT, Niehoff KE, Kalivas PW (2010). The infralimbic cortex regulates the consolidation of extinction after cocaine selfadministration. Learn Mem 17: 168-175.

Langton JM, Richardson R (2008). D-cycloserine facilitates extinction the first time but not the second time: an examination of the role of NMDA across the course of repeated extinction sessions. Neuropsychopharmacology 33: 3096-3102.

Leaderbrand K, Corcoran KA, Radulovic J (2014). Co-activation of NR2A and NR2B subunits induces resistance to fear extinction. Neurobiol Learn Mem 113: 35-40.

Ledgerwood L, Richardson R, Cranney J (2003). Effects of D-cycloserine on extinction of conditioned freezing. Behav Neurosci 117: 341-349.

Lee JL, Everitt BJ (2008). Appetitive memory reconsolidation depends upon NMDA receptor-mediated neurotransmission. Neurobiol Learn Mem 90: 147-154.

Lee JL, Milton AL, Everitt BJ (2006). Reconsolidation and extinction of conditioned fear: inhibition and potentiation. J Neurosci 26: 10051-10056.

Lehmann J, Schneider J, McPherson S, Murphy DE, Bernard P, Tsai C et al (1986). CPP, a selective N-methyl-D-aspartate (NMDA)type receptor antagonist: characterization in vitro and in vivo. J Pharmacol Exp Ther 240: 737-746.

Liu JL, Li M, Dang XR, Wang ZH, Rao ZR, Wu SX et al (2009). A NMDA receptor antagonist, MK-801 impairs consolidating extinction of auditory conditioned fear responses in a Pavlovian model. PLoS One 4: e7548.

Lozovaya NA, Grebenyuk SE, Tsintsadze T, Feng B, Monaghan DT, Krishtal OA (2004). Extrasynaptic NR2B and NR2D subunits of NMDA receptors shape 'superslow' afterburst EPSC in rat hippocampus. J Physiol 558: 451-463.

Mamiya N, Fukushima H, Suzuki A, Matsuyama Z, Homma S, Frankland PW et al (2009). Brain region-specific gene expression activation required for reconsolidation and extinction of contextual fear memory. J Neurosci 29: 402-413.

Matsuda S, Matsuzawa D, Nakazawa K, Sutoh C, Ohtsuka H, Ishii D et al (2010). D-serine enhances extinction of auditory cued fear conditioning via ERK1/2 phosphorylation in mice. Prog Neuropsychopharmacol Biol Psychiatry 34: 895-902.

McLamb RL, Williams LR, Nanry KP, Wilson WA, Tilson HA (1990). MK-801 impedes the acquisition of a spatial memory task in rats. Pharmacol Biochem Behav 37: 41-45.

McLellan AT, Lewis DC, O'Brien CP, Kleber HD (2000). Drug dependence, a chronic medical illness: implications for treatment, insurance, and outcomes evaluation. JAMA 284: $1689-1695$.

Mierzejewski P, Korkosz A, Rogowski A, Korkosz I, Kostowski W, Scinska A (2009). Post-session injections of a protein synthesis inhibitor, cycloheximide do not alter saccharin selfadministration. Prog Neuropsychopharmacol Biol Psychiatry 33: 286-289.

Millan EZ, Marchant NJ, McNally GP (2011). Extinction of drug seeking. Behav Brain Res 217: 454-462.

Miller CA, Marshall JF (2005). Molecular substrates for retrieval and reconsolidation of cocaine-associated contextual memory. Neuron 47: $873-884$.

Milton AL, Lee JL, Butler VJ, Gardner R, Everitt BJ (2008). Intraamygdala and systemic antagonism of NMDA receptors prevents the reconsolidation of drug-associated memory and impairs subsequently both novel and previously acquired drug-seeking behaviors. J Neurosci 28: 8230-8237.

Milton AL, Merlo E, Ratano P, Gregory BL, Dumbreck JK, Everitt BJ (2013). Double dissociation of the requirement for GluN2B- and GluN2A-containing NMDA receptors in the destabilization and restabilization of a reconsolidating memory. J Neurosci 33: 1109-1115.

Misanin JR, Miller RR, Lewis DJ (1968). Retrograde amnesia produced by electroconvulsive shock after reactivation of a consolidated memory trace. Science 160: 554-555.

Mondadori C, Weiskrantz L, Buerki H, Petschke F, Fagg GE (1989). NMDA receptor antagonists can enhance or impair learning performance in animals. Exp Brain Res 75: 449-456.

Nader K (2003). Memory traces unbound. Trends Neurosci 26: $65-72$.

Nader K, Schafe GE, Le Doux JE (2000). Fear memories require protein synthesis in the amygdala for reconsolidation after retrieval. Nature 406: 722-726.

Otis JM, Dashew KB, Mueller D (2013). Neurobiological dissociation of retrieval and reconsolidation of cocaineassociated memory. J Neurosci 33: 1271-1281a.

Otis JM, Fitzgerald MK, Mueller D (2014). Infralimbic BDNF/TrkB enhancement of GluN2B currents facilitates extinction of a cocaine-conditioned place preference. J Neurosci 34: 6057-6064.

Pedreira ME, Perez-Cuesta LM, Maldonado H (2002). Reactivation and reconsolidation of long-term memory in the crab Chasmagnathus: protein synthesis requirement and mediation by NMDAtype glutamatergic receptors. J Neurosci 22: 8305-8311.

Peters J, LaLumiere RT, Kalivas PW (2008). Infralimbic prefrontal cortex is responsible for inhibiting cocaine seeking in extinguished rats. J Neurosci 28: 6046-6053.

Pickens CL, Airavaara M, Theberge F, Fanous S, Hope BT, Shaham Y (2011). Neurobiology of the incubation of drug craving. Trends Neurosci 34: 411-420.

Quirk GJ, Mueller D (2008). Neural mechanisms of extinction learning and retrieval. Neuropsychopharmacology 33: 56-72.

Quirk GJ, Russo GK, Barron JL, Lebron K (2000). The role of ventromedial prefrontal cortex in the recovery of extinguished fear. J Neurosci 20: 6225-6231.

Sadler R, Herzig V, Schmidt WJ (2007). Repeated treatment with the NMDA antagonist MK-801 disrupts reconsolidation of memory for amphetamine-conditioned place preference. Behav Pharmacol 18: 699-703.

Santini E, Muller RU, Quirk GJ (2001). Consolidation of extinction learning involves transfer from NMDA-independent to NMDAdependent memory. J Neurosci 21: 9009-9017. 
Song C, Ehlers VL, Moyer JR Jr (2015). Trace fear conditioning differentially modulates intrinsic excitability of medial prefrontal cortex-basolateral complex of amygdala projection neurons in infralimbic and prelimbic cortices. J Neurosci 35: 13511-13524.

Sotres-Bayon F, Diaz-Mataix L, Bush DE, LeDoux JE (2009). Dissociable roles for the ventromedial prefrontal cortex and amygdala in fear extinction: NR2B contribution. Cereb Cortex 19: 474-482.

Stern CA, Gazarini L, Vanvossen AC, Hames MS, Bertoglio LJ (2014). Activity in prelimbic cortex subserves fear memory reconsolidation over time. Learn Mem 21: 14-20.

Suzuki A, Josselyn SA, Frankland PW, Masushige S, Silva AJ, Kida S (2004). Memory reconsolidation and extinction have distinct temporal and biochemical signatures. J Neurosci 24: 4787-4795.

Thompson DM, Winsauer PJ, Mastropaolo J (1987). Effects of phencylidine, ketamine, and MDMA on complex operant behavior in monkeys. Pharmacol Biochem Behav 26: 401-405.
Torras-Garcia M, Lelong J, Tronel S, Sara SJ (2005). Reconsolidation after remembering an odor-reward association requires NMDA receptors. Learn Mem 12: 18-22.

Tronson NC, Taylor JR (2007). Molecular mechanisms of memory reconsolidation. Nat Rev Neurosci 8: 262-275.

Walker DL, Ressler KJ, Lu KT, Davis M (2002). Facilitation of conditioned fear extinction by systemic administration or intraamygdala infusions of D-cycloserine as assessed with fearpotentiated startle in rats. $J$ Neurosci 22: 2343-2351.

Wells AM, Xie X, Higginbotham JA, Arguello AA, Healey KL, Blanton $M$ et al (2016). Contribution of an SFK-mediated signaling pathway in the dorsal hippocampus to cocainememory reconsolidation in rats. Neuropsychopharmacology 41: 675-685.

Wouda JA, Diergaarde L, Riga D, van Mourik Y, Schoffelmeer AN, De Vries TJ (2010). Disruption of long-term alcohol-related memory reconsolidation: role of beta-adrenoceptors and NMDA Receptors. Front Behav Neurosci 4: 179.

Supplementary Information accompanies the paper on the Neuropsychopharmacology website (http://www.nature.com/npp) 